no questions where HealthRota was inferior to other rotas. The biggest improvements were seen with 'Requesting Leave', 'Access the rota 24 hours a day' and 'Feeling of Ownership'. There were many positive comments in the open section including HealthRota was 'Much less Stressful', 'Absolutely fantastic' and 'A breath of fresh air'.

Conclusions Despite COIVD-19 impacting on the junior doctor experience. HealthRota still proved to be a much more popular and efficient system across all important aspects of medical rotas

\section{INFLIXIMAB INDUCED INTERSTITIAL LUNG DISEASE AND RESPIRATORY FAILURE:CASE REPORT OF A CHILD WITH INFLAMMATORY BOWEL DISEASE}

Anna Evans, Thomas Cromarty, Benjamin Eagle, Michael Cosgrove, Amar Wahid, Atheer Abdulameer. UK

10.1136/bmjpo-2021-RCPCH.71

Background Infliximab is a chimeric anti-tumour necrosis factor-alpha (anti-TNFa) monoclonal antibody that has been used safely in the management of Inflammatory Bowel Disease (IBD) for over 20 years, recommended for use in paediatrics by the National Institute for Clinical Excellence (NICE) since 2010. Drug-induced interstitial lung disease (DIILD) secondary to Infliximab has been described as a rare complication in adult literature with variable outcomes.

Objectives We present the first reported case of life-threatening respiratory disease in a 13 year old patient receiving Infliximab for IBD.

Methods The patient was diagnosed with unclassified IBD (IBDU) aged 11 years, when he presented with colitis. Following steroid induction, maintenance treatment with Azathioprine was not tolerated and response to Mercaptopurine was inadequate. There was complete resolution of symptoms after the third does of Infliximab.

Following the fourth dose of Infliximab the patient presented with respiratory symptoms which progressed rapidly, requiring Paediatric Intensive Care Unit (PICU) admission. Treatment was directed at a presumed infectious cause. CRP was raised and the patient became febrile, however extensive microbiological investigations were negative.

On day 8 of admission Methylprednisolone was started. Clinical improvement was rapid and allowed weaning of organ support. At day 14 Methylprednisolone dose was reduced by half and antibiotics were discontinued. Rapid deterioration ensued, therefore Methylprednisolone was increased.

After 5 weeks of intensive organ support and $2 \mathrm{mg} / \mathrm{kg} / \mathrm{day}$ of methylprednisolone, the patient improved and steroids were cautiously weaned. Respiratory symptoms had completely resolved 8 weeks after last receiving Infliximab.

Subsequently he had a recurrence of symptoms with colitis confirmed on colonoscopy, and histology more in keeping with Crohn's disease. He did not respond to 5ASA therapy. After MDT discussion he was treated with Vedolizumab (a gut-specific anti-integrin). His colitis settled, with no adverse reaction to the alternative biologic.

Results Studies into DIILD from biologic therapies are largely from Rheumatoid Arthritis (RA) cohorts, and many subjects had pre-existing ILD. Known risk factors for the development of, and mortality from DIILD are not relevant to this case. A diagnosis of DIILD secondary to Infliximab was concluded based on clinical course, lack of alternative diagnoses after thorough investigation, and multi-disciplinary discussions.

Withdrawal of the causative drug is the universally accepted treatment. Steroids are the most frequent drug treatment used but optimal dosing, duration and patient group who may benefit is unclear. Our case supports the use of high dose steroids in severely unwell patients but limited evidence precludes recommendation of other drug treatments.

Conclusions We believe this is the first case report of a child with DIILD secondary to Infliximab. Withdrawal of Infliximab and treatment with high dose steroids during the period of drug clearance was life-saving. Identification of those at risk of DIILD and early detection of the disease is challenging; there are many confounding factors and DIILD appears to be very rare in paediatrics. We would advocate further research to develop validated biomarkers for early detection of DIILD. Reporting of adverse events in post-marketing era remains integral. Vedolizumab may be an appropriate alternative for patients who have adverse reactions to Infliximab.

\section{THE OUTCOME OF PDA LIGATION BY MINI- THORACOTOMY IN PREMATURE NEONATES: A SINGLE HOSPITAL EXPERIENCE}

Zainab Irshad, Noor Alhashimi Alhashimi, Saeed Al Hindi. Bahrain

\subsection{6/bmjpo-2021-RCPCH.72}

Background Patent ductus arteriosus (PDA) is a common complication observed in the premature infant; its management being a controversial issue. The lack of robust evidence of benefit or harms of available treatment options, necessitate the need to balance them out against the likelihood of spontaneous duct constriction, which occurs in approximately a third of extremely premature neonates. Despite the controversy regarding the optimal management of PDA, nearly $70 \%$ of preterm infants (less than 28 weeks of gestation) will receive either medical or surgical treatment. The rationale behind this is to decrease the likelihood of developing morbidities associated with prematurity and persistent PDA. These include intraventricular hemorrhage (IVH), bronchopulmonary dysplasia, necrotizing enterocolitis (NEC) and increased mortality.

Various thoracotomy practices have been employed for occlusion of PDA which are not amenable to medical management. Success rates of surgical ligation are reported to be high but it has been associated with significant operative complications. In this study, we assess survival outcomes and operative complications associated with ligation.

Objectives This prospective study focuses on the closure of PDA by mini-thoracotomy in our institution. We report our preliminary experience of using this approach in small premature infants and determining survival outcomes in relation to factors such as gender, birth weight, age, and type of ventilation used intra-operatively.

Methods Between January 2004 and December 2012, 52 consecutive premature infants with an echocardiographic diagnosis of isolated PDA, which are not amenable to medical treatment, were included. Those with chromosomal abnormalities, major cardiac congenital anomalies aside from septal defects, and infants who did not receive mechanical ventilation in the first week of life were excluded. The median gestational age was 28 weeks and the median gestational weight at surgery was $705 \mathrm{~g}$. The median PDA size was $3.8 \mathrm{~mm}$, ranging from 